\title{
Performance Evaluation of the Striped Checkpointing Algorithm on the Distributed RAID for Cluster Computer
}

\author{
Yun Seok Chang ${ }^{1}$, Sun Young $\mathrm{Cho}^{2}$, and Bo Yeon Kim ${ }^{3}$ \\ 1 Department of Computer Engineering, Daejin University, Pocheon, Korea \\ 2 Basic Science Research Institute, Chungbuk University, Chungju, Korea \\ 3 BK21 Department of Electrical and Computer Engineering, Kangwon National \\ University Chuncheon, Korea
}

\begin{abstract}
The distributed RAID for serverless cluster computer is used to save the checkpoint files periodically according to the checkpointing algorithm for rollback recovery. Striped checkpointing algorithm newly proposed in this paper can adopt the merits of the coordinated and the staggered checkpointing algorithms. Coordinating enables parallel $\mathrm{I} / \mathrm{O}$ on distributed disks and staggering avoids network bottleneck in distributed disk I/O operations. With a fixed cluster size, we reveal the tradeoffs between these two speedup techniques. The striped checkpointing approach allows dynamical reconfiguration to minimize checkpointing overhead among concurrent software processes. We demonstrate how to reduce the overhead by striping and staggering dynamically. For communication-intensive computational programs, this new scheme can significantly reduce the checkpointing overhead. Linpack HPC Benchmark results prove the benefits of trading between stripe parallelism and distributed staggering. These results are useful to design efficient checkpointing algorithm for fast rollback recovery from any single node failure in a cluster computer.
\end{abstract}

\section{Introduction}

In the typical cluster computer system, all concurrent processes on different nodes should communicate with each other through message passing. Checkpointing algorithm can maintain the global consistency among the nodes by saving the checkpoint data to the stable storage. Several checkpointing algorithms have been studied on the cluster computer. Coordinated checkpointing [1] takes checkpoints from each node simultaneously at every time interval to yield a consistent recovery globally in case of any failure. The drawback of the coordinated checkpointing is in the loss of freeze time for collecting checkpoints from nodes at the same time and heavy network created by synchronized checkpointing. Simultaneous writing of checkpoint files into local disks may cause a heavy network contention problem and disk IO bottleneck. To solve the disk contention problem, diskless checkpointing algorithm was introduced[6]. But the fault coverage is rather limited in a diskless checkpointing algorithm. So, 
staggered checkpointing algorithm was introduced to write checkpoints onto a central stable storage 8 . It can greatly reduce the storage contention to some extent. But the staggered checkpointing algorithm has to pay additional overhead to achieve correct rollback recovery to avoid inconsistent states among cluster nodes. Therefore, if the size of the cluster increases, message logging overhead also increases. In a serverless cluster system, the distributed RAID system is suitable for saving distributed checkpoint files simultaneously in disks attached to cluster nodes 4]. Based on the distributed RAID, we propose striped checkpointing scheme. This scheme has two advantages. First, simultaneous distribution of the checkpoint files greatly alleviates the network IO contention problem by taking advantage of parallel IO. Second, rollback recovery latency is reduced on a distributed RAID system. So, mean time to repair of cluster is reduced and high cluster availability can be achieved. To take advantage of parallelism of the distributed RAID, the staggered checkpointing algorithm can be combined with striped checkpointing algorithm. Only a subset of disks constitutes a stripe group to take the checkpoint simultaneously. After one stripe group finishes checkpointing, another stripe group starts checkpointing in a staggered manner. This striped checkpointing algorithm has two benefits. First, when the IO contention is more serious a problem, parallel IO of striped group helps lessening the problem. Second, if network contention dominates, staggering can reduce it. To evaluate the performance of the striped checkpointing scheme, we carried out benchmark tests and compared with other checkpointing algorithm.

\section{Distributed RAID and Single IO Space}

To build a distributed RAID, all disks embedded in the cluster must establish the single IO space(SIOS). The SIOS should have high scalability, availability, and compatibility with IO centric cluster application. These should also imply a total transparency to the users, who can utilize all disks without knowing the physical locations of the data blocks. On NFS of centralized cluster file server, all checkpoint files are saved on a network file system. On SIOS, checkpoint files spread over the distributed disks. At the striped checkpointing scheme, checkpoint files are saved over the distributed RAID system. Distributed RAID is crucial to building scalable cluster of computers [5], and SIOS can be implemented by using cooperative device drivers working at the system kernel[3]. These drivers work together to establish the SIOS across all physical distributed disks. Once the SIOS is established, all disks will be used collectively as a single global virtual disk. Then cluster can be built serverless and offers remote disk access directly at the kernel level. Parallel IO is made possible on any subset of local disks, since all distributed disks form SIOS. So, no heavy cross-node or process system calls are needed to perform remote file access. 


\section{Striped Checkpointing Scheme}

The original concept of staggered checkpointing allows only one process to store the checkpoint at a time. Figure1 1 shows the timing diagram of staggered checkpoint scheme. Although this scheme introduces non-blocking, lessen IO and network contention, it suffers from message logging 2 overhead and many control messages to avoid the inconsistency problem among nodes. Therefore, staggered checkpointing scheme increases the checkpoint overhead dramatically according to the cluster size and causes the traffic for stable storage to save message log information. Simultaneous writing of multiple processes in coordinated checkpointing also causes the network contention and IO bottleneck problem as the cluster size is increased. Parallel IO capacity of a distributed RAID is applied to

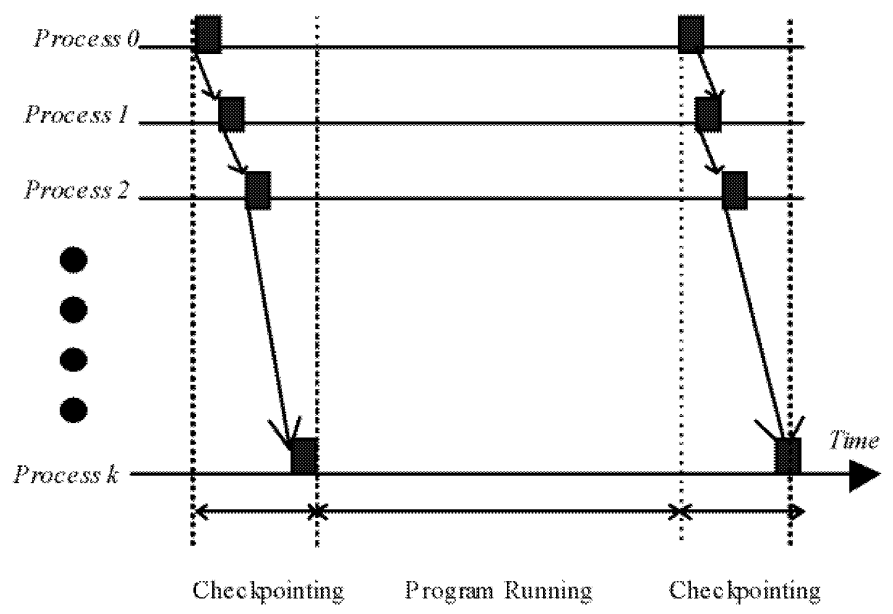

Fig. 1. Staggered checkpointing scheme

achieve fast checkpointing in a cluster system. The striped checkpointing scheme saves the checkpoint files over distributed disks that forming a stripe group. A stripe group is defined as a subset of disks that can be accessible in parallel. Only the disks in the same stripe group can initiate checkpointing simultaneously. To alleviate the network contention, staggered writing method is combined with the striped groups. Figure 2 shows this concept clearly. On the striped checkpointing scheme, each stripe group takes its checkpoints one after another. The stripe group leads to parallelism and avoids IO contention of the storage. This scheme can enhance both network utilization and IO performance. There exists a tradeoff between stripe parallelism and staggering depth. One can reconfigure the relative stripe size and staggering depth to achieve maximum benefits from it on a particular application. Higher parallelism can lead higher aggregate disk 


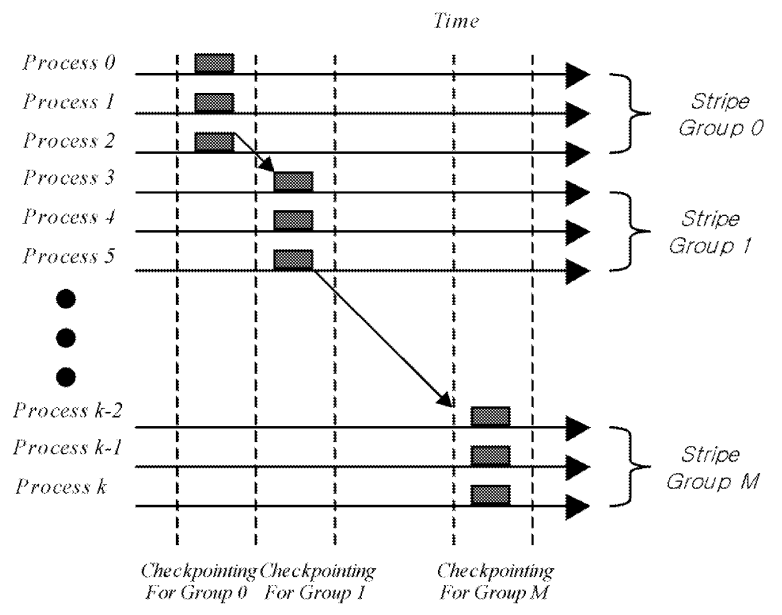

Fig. 2. Striped checkpointing scheme

bandwidth and higher staggering depth can lessen the contention problem with the least cost of inconsistency.

\section{Performance Evaluation Environment}

To evaluate the performance of the striped checkpointing scheme and to compare with other schemes, we choose Linpack HPC Benchmak to carry out the massive computational cluster experiments with MPI(Message Passing Interface). The size of checkpoint data is used to be proportional to the problem size of the computational program. The library function program Libckpt [7] is inserted in Linpack HPC computational program to implement a full-state, non-incremental checkpoint with the least system load in the Linux cluster environment. The performance is directly indicated by the checkpoint overhead and IO throughput. Our cluster platform is configured as 16 system nodes connected through the 100Mbps dedicated ethernet switch. Each node consists of Pentium-II processor, $128 \mathrm{MB}$ main memory, a 4GB local disk in single partition with Redhat Linux 6.2. Two storage schemes are used for saving checkpoints. On NFS, checkpoints of all nodes are saved only one disk on a dedicated node. On SIOS, 8 disks among the 16 disks are participated in the SIOS scheme. In our experiments, three checkpointing schemes, coordinated checkpoint, staggered checkpoint and striped checkpoint, are conducted on benchmark program. The coordinated checkpoint scheme forces all processes to take checkpoints at the same time. Although the cluster size would not be enough to show the drawback of the coordinated checkpointing scheme, we show the performance just to compare it's relative performance with staggered checkpointing scheme. The staggered checkpoint scheme allows only one node takes checkpoint at a time. 
The striped checkpoint scheme takes three stripe configurations: 2, 4, and 8 disks in a striped group. The computational problem size of Linpack HPC Benchmark is varied from 1000 to 5000 to drive various workloads in our cluster system like light, medium and heavy workload reaches from 2.3 to $12.1 \mathrm{Gflops}$ during experiments.

\section{Result and Discussion}

Through the performance evaluation in various configuration, we had lots of impressive results. Figure 3 and 4 show the checkpointing overheads vs. problem size for all checkpointing schemes on NFS and 8 disks SIOS configuration. Checkpointing time represents total IO time including checkpoint file saving time, message logging time, and other communication overheads. Figure 5 and [6] also show the IO throughput for all checkpointing algorithm on NFS and SIOS configuration. IO throughput represents data transfer rate from nodes to NFS file server or distributed RAID system for checkpointing files. The results show that coordinated checkpointing scheme has the best performance in checkpointing time and IO throughput because of the small cluster size and parallel IO operation of the distributed RAID at first. But coordinated checkpointing will cause serious network contention when the cluster size in increases enough to over the network capacity. So, it makes sense to compare striped checkpointing scheme with staggered checkpointing scheme under our cluster platform.

\section{Ckeckpointing time at NFS}

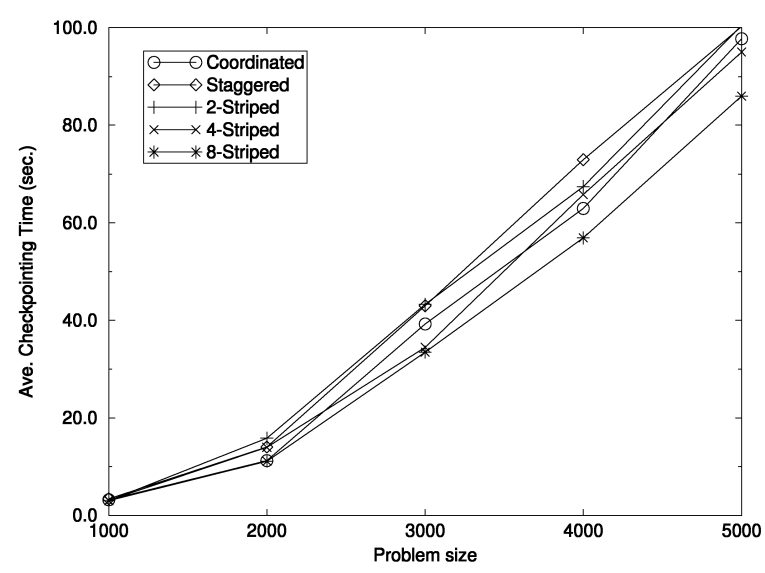

Fig. 3. Checkpointing overhead on NFS 


\section{Ckeckpointing time at 8-disks SIOS}

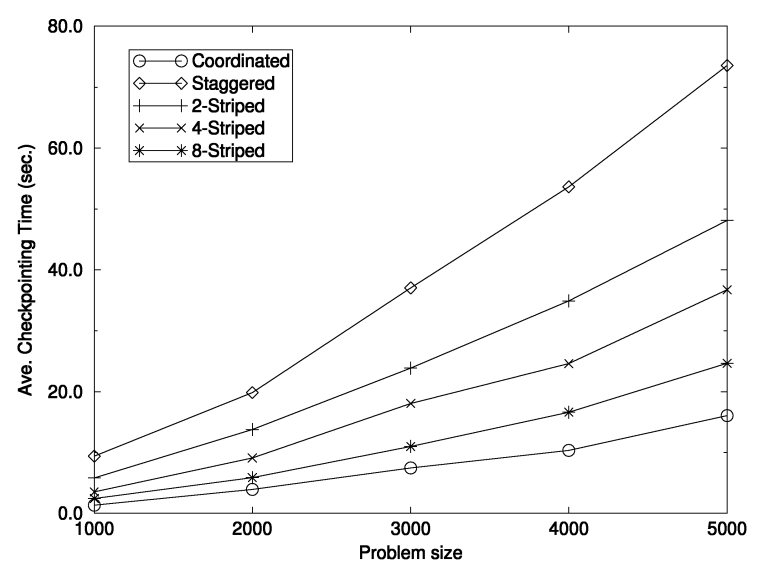

Fig. 4. Checkpointing overhead on SIOS

On NFS, all checkpointing schemes have not so much difference among their checkpointing time and throughput. It shows that IO contention on the cluster file server dominates over network contention. Therefore, checkpointing schemes do not make much sense on the checkpointing performance on NFS. Results on SIOS show the effect of the distributed RAID system results in its parallel IO operation. On SIOS, striped checkpointing scheme has better performance than staggered checkpointing scheme. Moreover, larger stripe group size leads to better performance until parallel IO bandwidth of a stripe group does not exceed total network capacity. On our cluster platform, 8-striped checkpointing scheme shows over 4 times better checkpointing time and over 3 times IO throughput than staggered checkpointing scheme. In the Linpack HPC Benchmark, large problem size generates a large checkpoint file. It results in performance gap between staggered checkpointing scheme and striped checkpointing scheme according to the problem size in checkpointing time.

The IO throughput of the striped checkpointing schemes are slightly down at the high problem size because of the IO bottleneck at distributed RAID system and still better performance than staggered checkpointing scheme. These results prove that maximum size of stripe group within the network bandwidth can produce the best performance on the distributed RAID system with SIOS although the cluster size becomes very large enough to stall network when coordinated checkpointing scheme is applied. This means that striped checkpointing scheme needs some negotiation between network bandwidth and size of stripe group. Therefore, a cluster system designed with ate size of stripe group will pay the least overhead for checkpointing operation under 


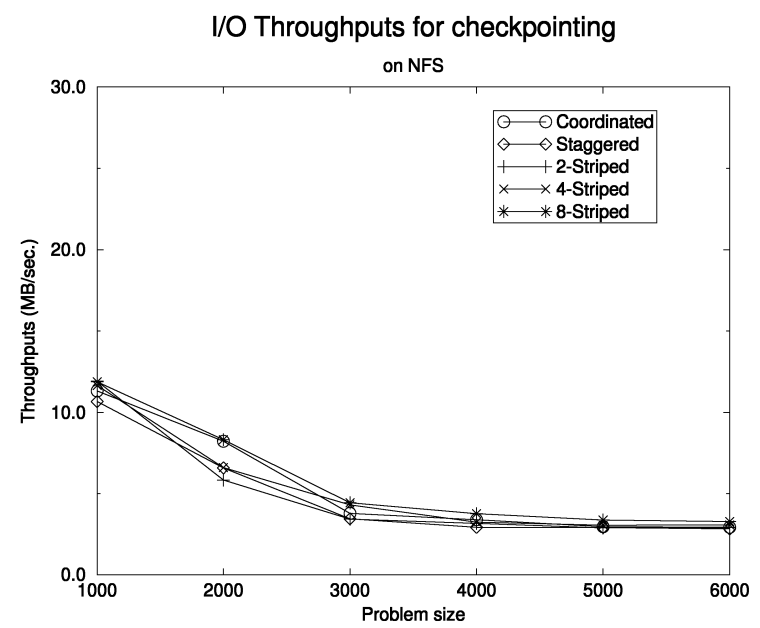

Fig. 5. Throughput on NFS

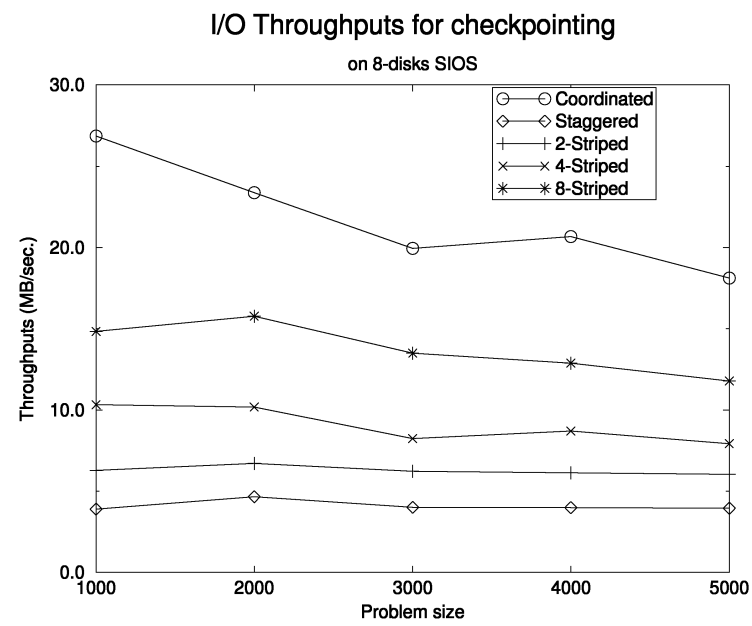

Fig. 6. Throughput on SIOS

the striped checkpointing scheme. If the cluster size is increased, we can easily guess the checkpointing overhead and the throughput would show something different result. We are going to deal the correlation between cluster size and rollback recovery performance with heavier computational problem at the further research. 


\section{Conclusion}

The striped checkpointing scheme has been shown effective to perform checkpointing on cluster with SIOS. Striping exploits parallelism across multiple disks. Staggering alleviates the IO bottleneck on each disk. Therefore, striped checkpointing scheme can show better performance than other checkpointing schemes when the cluster size becomes large enough to over the IO and network bandwidth limitation. The tradeoff between striping and staggering should be applied dynamically for different applications. The tradeoff results can be applied to yield the striped checkpointing configuration with the lowest cost for a given application. Higher stripe parallelism can lead to higher aggregate disk bandwidth and higher staggering depth copes better with the network contention problem. Therefore, the stripe size suitable on a particular application should be established to get the best checkpointing performance on the distributed RAID system.

\section{References}

1. Cao, G., Singhal, M. : On Coordinated Checkpointing in Distributed Systems. IEEE Transactions on Parallel and Distributed Systems. 9(12) (1998)

2. Elnozahy, E., Zwaenepoel, W. : On the Use and Implementation of Message Logging. Proceedings of 24th International Symposium on Fault-Tolerant Computing. (1994)

3. Hwang, K., Jin, H., Ho, R. : RAID-x: A New Distributed Disk Array for I/O-Centric Cluster Computing. Proceedings of 9th High-Performance Distributed Computing Symposium. (2000)

4. Hwang, K., Jin, H., Ho, R., Ro, W. : Reliable Cluster Computing with a New Checkpointing RAID-x Architecture. Proceedings of 9-th Workshop on Heterogeneous Computing. (2000)

5. Hwang, K., Jin, H., Chow, E., Wang, C., Xu, Z. : Designing SSI Clusters with Hierarchical Checkpointing and Single IO Space. IEEE Concurrency Magazine. (1999)

6. Plank, J., Li, K., Puening, M. : Diskless Checkpointing. IEEE Transactions on parallel and Distributed Systems. (1998)

7. Plank, J., Beck, M., Kingsley, G., Li, K. : Libckpt: Transparent Checkpointing Under UNIX. Proceedings of USENIX Winter 1995 Technical Conference. (1995)

8. Vaidya, N. : Staggered Consistent Checkpointing. IEEE Transactions on Parallel and Distributed Systems. 10(7) (1999) 